\title{
MÉTODO BOOTSTRAP PARA HIPÓTESIS CONCERNIENTES A LA DIFERENCIA DE MEDIAS PARA MUESTRAS PAREADAS: APLICACIONES
}

\section{Bootstrap Method for Hypotheses Concerning the Difference of Means for Paired Samples: Applications}

\author{
${ }^{1}$ Antonio Meneses* ${ }^{2}$ Lourdes Zuñiga, ${ }^{2}$ Carlos Santos, ${ }^{2}$ Silvia Haro, \\ ${ }^{2}$ Nancy Chariguamán ${ }^{2}$ Luis Vera \\ ${ }^{1}$ Universidad Nacional de Chimborazo, Riobamba, Ecuador \\ ${ }^{2}$ Escuela Superior Politécnica de Chimborazo, Riobamba, Ecuador \\ *ameneses@unach.edu.ec
}

\section{$R$ esumen}

En este trabajo se propone un método flexible para calcular un intervalo de confianza con réplicas Bootstrap para la diferencia de medias de dos muestras pareadas sin necesidad de normalidad, en el ámbito de la teoría de hipótesis. La hipótesis nula se rechaza si el intervalo de confianza no contiene el 0 , caso contrario se acepta. Dos aplicaciones se realizan con las variables: producción mensual de leche y producción promedio mensual de leche, de vacas alimentadas solo con forraje y con forraje más ensilaje de maíz. También se realiza un estudio de simulación con muestras normales que se obtienen resultados similares con el método comúnmente utilizado de la t-Student y el método propuesto Bootstrap.

Palabras claves: método bootstrap, diferencias-medias, muestras pareadas.

\section{A bstract}

In this paper, we propose a flexible method to calculate a confidence interval with bootstrap replicas for the mean difference of two paired samples without the need of normality, in the field of hypothesis theory. The null hypothesis is rejected if the confidence interval does not contain the 0 , otherwise it is accepted. Two applications are made with the variables : monthly milk production and average monthly milk production, from cows fed only with forage and with forage plus corn silage. A simulation study is also carried out with normal samples, obtaining similar results with the com- monly used method of t-Student and the proposed Bootstrap method.

Keywords: bootstrap method, differences-means, paired samples.

\section{INTRODUCCIÓN}

Los métodos paramétricos comunes para la diferencia de medias en el contexto de la teoría de hipótesis tienen condiciones fuertes que deben cumplir las variables, una de ellas es la normalidad (3). El método que se propone es muy flexible en cuanto a que las variables sean normales o no. Este método basado en Bootstrap $(1,2)$, principalmente se centra en calcular un 
intervalo de confianza para la diferencia de medias de dos muestras pareadas de acuerdo a lo siguiente:

Primero, se realiza la parte teórica propuesta en forma de algoritmo con 9 pasos en los que se detalla claramente la manera de calcular el intervalo de confianza Bootstrap para la diferencia de medias de dos muestras pareadas.

Segundo, se presentan los lineamientos para seleccionar la hipótesis nula, notación para las hipótesis (3). También se plantea de manera sistemática la prueba de hipótesis en cinco pasos haciendo énfasis en el método Bootstrap propuesto. Tercero, se realizan los resultados y las discusiones al aplicar los cinco pasos de la prueba de hipótesis usando el intervalo de confianza Bootstrap al $95 \%$ para la diferencia de medias de dos muestras pareadas. También se realiza un estudio de simulación con muestras normales mediante la función rnorm del software $\mathrm{R}$ (6), obteniendo resultados similares con el método comúnmente utilizado de la t-Student y el método propuesto Bootstrap.

Por último, se dan las conclusiones del trabajo.

\section{Intervalo de confianza Bootstrap en hipótesis concernientes para la diferencia de medias en muestras pareadas.}

El método Bootstrap es un procedimiento estadístico que sirve para aproximar la distribución en el muestreo (normalmente de un estadístico). Para ello se procede mediante remuestreo, es decir, obteniendo muestras mediante algún procedimiento aleatorio que utilice la muestra original (2). sobre la base de los aspectos generales de este método y sin dejar de lado la hipótesis nula, se calcula el intervalo de confianza para la diferencia de medias de dos muestras pareadas usando los pasos del siguiente algoritmo:
1. Dada la muestra pareada:

$$
(\mathrm{X}, \mathrm{Y})=\left\{\left(\mathrm{X}_{1}, \mathrm{Y}_{1}\right), \ldots,\left(\mathrm{X}_{\mathrm{n}}, \mathrm{Y}_{\mathrm{n}}\right)\right\}
$$

crear la muestra de diferencias:

$$
\mathrm{D}=\left\{\mathrm{X}_{1}-\overline{\mathrm{X}}-\mathrm{Y}_{1}+\overline{\mathrm{Y}}, \ldots, \mathrm{X}_{\mathrm{n}}-\overline{\mathrm{X}}-\mathrm{Y}_{\mathrm{n}}+\overline{\mathrm{Y}}\right\}
$$

2. Para cada $i=1,2, \ldots, n$ arrojar

$$
\mathrm{U}_{\mathrm{i}} \sim \mathrm{U}(0,1) \text { y hacer } \mathrm{D}_{\mathrm{i}}^{*}=\mathrm{D}_{[\mathrm{nUi}]+1}
$$

3. Obtener $\overline{\mathrm{D}^{*}}=\frac{1}{\mathrm{n}} \sum \mathrm{D}_{\mathrm{i}}^{*}$

$$
\mathrm{S}_{\mathrm{D}^{*}}^{2}=\frac{1}{\mathrm{n}-1} \sum\left(\mathrm{D}_{\mathrm{i}}^{*}-\overline{\mathrm{D}^{*}}\right)^{2}
$$

4. Calcular el estadístico bootstrap:

$$
\mathrm{R}^{*}=\frac{\overline{\mathrm{D}^{*}}}{\frac{\mathrm{S}_{\mathrm{D}^{*}}}{\sqrt{\mathrm{n}}}}
$$

5. Repetir B veces los pasos 2, 3 y 4 para obtener las B réplicas bootstrap de $\mathrm{R}^{*}$. Luego ordenar de forma creciente estas réplicas del modo que:

$$
\mathrm{R}_{1}^{*} \leq \mathrm{R}_{2}^{*} \leq \ldots \leq \mathrm{R}_{\mathrm{B}}^{*}
$$

6. Calcular los puntos críticos, inferior y superior al nivel de significancia $\alpha$ :

$$
\begin{gathered}
\text { p.c.inf }=\mathrm{R}_{\left[\mathrm{B} \frac{\alpha}{2}\right]}^{*} \\
\text { p.c.sup }=\mathrm{R}_{\left[\mathrm{B}\left(1-\frac{\alpha}{2}\right)\right]}^{*}
\end{gathered}
$$

7. Calcular los límites inferior y superior del intervalo de confianza para el nivel de significancia $\alpha$ (ver detalles en el numeral 4 de la prueba de hipótesis):

$\lim \cdot \inf =$

$$
\overline{\mathrm{DIF}} \text { - p.c.sup } \sqrt{\frac{\mathrm{S}_{\mathrm{DIF}}^{2}}{\mathrm{n}}}
$$

Lim.sup $=$

$$
\overline{\mathrm{DIF}}-\text { p.c.inf } \sqrt{\frac{\mathrm{S}_{\mathrm{DIF}}^{2}}{\mathrm{n}}}
$$


Donde DIF $=\mathrm{X}-\mathrm{Y}, \overline{\mathrm{DIF}}=\overline{\mathrm{X}-\mathrm{Y}}, \mathrm{S}_{\mathrm{DIF}}^{2}$ es la desviación típica muestral, [[·]] la función parte entera y $\mathrm{U}(0,1)$ es la distribución uniforme en el intervalo $(0,1)$.

\section{Hipótesis nulas y prueba de hipótesis para diferencia de medias en muestras pareadas (3)}

\section{- Lineamiento para seleccionar la hipótesis nula:}

Cuando la meta de un experimento sea establecer una afirmación, la negación de la afirmación debería tomarse como la hipótesis nula. La afirmación se convierte en la hipótesis alternativa.

Notación para las hipótesis:

$\mathrm{H}_{0}$ : hipótesis nula

$\mathrm{H}_{1}$ : hipótesis alternativa.

\section{- Prueba de hipótesis:}

Para tratar sistemáticamente los problemas de pruebas de hipótesis, será útil proceder como se bosqueja en los siguientes cinco pasos (3):

1. Formular una hipótesis nula $\left(\mathrm{H}_{0}\right)$ y una hipótesis alternativa $\left(\mathrm{H}_{1}\right)$ adecuada que se acepte cuando deba rechazarse la hipótesis nula. Estadísticamente se procede a considerar la muestra de las diferencias:

$$
\mathrm{DIF}=\mathrm{X}-\mathrm{Y}
$$

Esta colección de diferencias se trata como una muestra aleatoria de tamaño $\mathrm{n}$ de una población que tiene media $\mu \_$DIF. Donde $\mu \_$DIF $=0$ se interpreta como indicador de que las medias de las dos variables son las mismas (hipótesis nula), así como $\mu \_D I F \neq 0$ como indicador de que la media de la variable $X$ es diferente que la media de la variable $\mathrm{Y}$ (hipótesis alternativa).

\section{Especificar el nivel de significancia $\alpha$.}

3. Construir un criterio para poner a prueba la hipótesis nula contra la alternativa dada.

4. A partir de los datos, calcular el intervalo de confianza con el algoritmo Bootstrap propuesto basado en la razón:

$$
\mathrm{R}=\frac{\overline{\mathrm{DIF}}-\mu_{\mathrm{DIF}}}{\frac{\mathrm{S}_{\mathrm{DIF}}}{\sqrt{\mathrm{n}}}}
$$

donde los límites del intervalo de confianza para $\mu_{\mathrm{DIF}}$ esta dado:

$$
\mathrm{R}_{\mathrm{inf}}<\frac{\overline{\mathrm{DIF}}-\mu_{\mathrm{DIF}}}{\frac{\mathrm{S}_{\mathrm{DIF}}}{\sqrt{\mathrm{n}}}}<\mathrm{R}_{\text {sup }}
$$

$\mathrm{R}_{\text {inf }} \mathrm{y} \mathrm{R}_{\text {sup }}$ son estimados con el algoritmo Bootstrap propuesto obteniendo:

$$
\text { p.c.inf. }<\frac{\overline{\mathrm{DIF}}-\mu_{\mathrm{DIF}}}{\frac{\mathrm{S}_{\mathrm{DIF}}}{\sqrt{\mathrm{n}}}}<\text { p.c.sup }
$$

A continuación despejando $\mu_{\mathrm{DIF}}$ sus límites del intervalo de confianza de son:

Límite inferior:

$$
\overline{\mathrm{DIF}} \text { - p.c.sup } \frac{\mathrm{S}_{\mathrm{DIF}}}{\sqrt{\mathrm{n}}}
$$

Límite superior:

$$
\overline{\mathrm{DIF}} \text { - p.c.inf } \frac{\mathrm{S}_{\mathrm{DIF}}}{\sqrt{\mathrm{n}}}
$$

5. Decidir si hay que rechazar la hipótesis nula o fallar en rechazarla, al verificar que el cero no pertenece o pertenece al intervalo de confianza de $\mu$ _DIF respectivamente.

\section{RESULTADOS Y DISCUSIÓN}

Fuente de datos:

El desarrollo del trabajo se realiza con dos variables: producción mensual de leche $\mathrm{y}$ producción promedio mensual de leche, en vacas alimentadas solo con forraje y con forraje más ensilaje de maíz en los meses de julio y agosto respectivamente. Estas muestras son tomadas en la Estación Experimental Tunshi, Facultad de Ciencias Pecuarias de la Escuela Superior Politécnica de Chimborazo, Riobamba, Ecuador. 


\section{Aplicaciones de inferencias concernientes a la diferencia de medias en prueba de hipótesis para muestras pareadas}

- Diferencia de medias entre las muestras pareadas:

X: producción de leche en litros de 20 vacas alimentadas solo con forraje en el mes de julio de 2017.

Y: producción de leche en litros de 20 vacas alimentadas con forraje más ensilaje de maíz en el mes de agosto de 2017.

Prueba de hipótesis:

1. $\mathrm{H}_{0}: \mu_{\mathrm{DIF}}=0$ (No existe diferencia significativa entre las medias de las variables $\mathrm{X}$ e $\mathrm{Y}$ )

$\mathrm{H}_{1}: \mu_{\mathrm{DIF}} \neq 0$ (Sí existe diferencia significativa entre las medias de las variables $\mathrm{X}$ e $\mathrm{Y}$ )

2. Nivel de significancia $\alpha=0,05$

3. El criterio de aceptar la hipótesis nula es cuando el 0 pertenece al intervalo de confianza Bootstrap, caso contrario la hipótesis nula es rechazada.

4. El intervalo de confianza Bootstrap al $95 \%$ es calculado siguiendo los pasos del algoritmo del método Bootstrap propuesto (con 1000 réplicas):

5. Decisión: en la Figura 3 se observa que el intervalo de confianza bootstrap no contiene el 0 , por lo que la hipótesis nula se rechaza, es decir que las medias de las variables $\mathrm{X}$ e $\mathrm{Y}$ son significativamente diferentes al 95\% de confianza. También se observa que el intervalo de confianza contiene únicamente valores positivos, que significa que la media de producción de leche en vacas alimentadas solo con forraje es significativamente mayor a la media de producción de leche en vacas alimentadas con forraje más ensilaje de maíz.

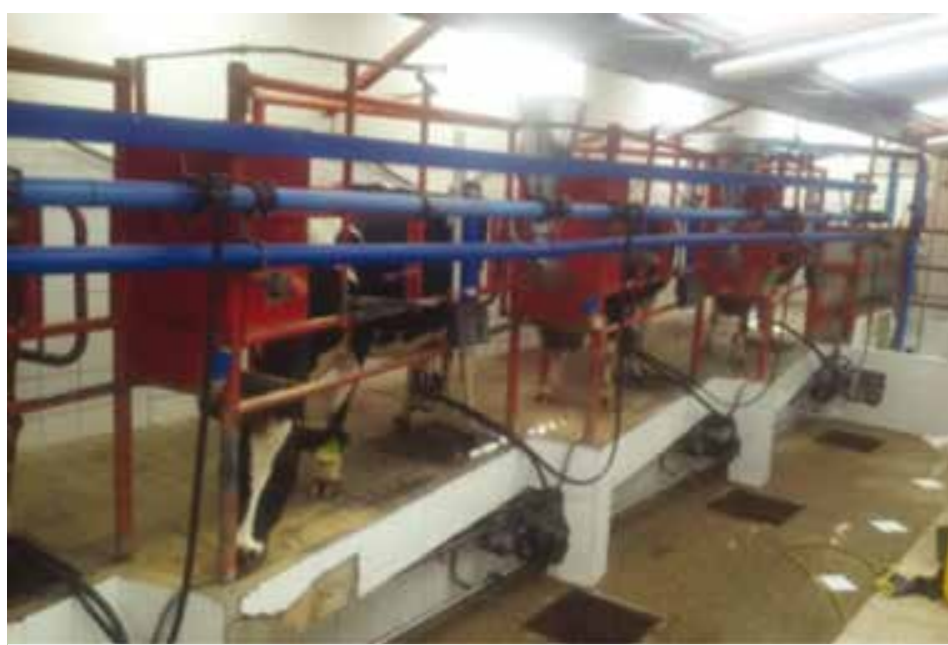

Figura 1: Unidad Académica y de Investigación en Bovinos Lecheros de la Estación Experimental Tunshi (foto tomada por el Ingeniero Carlos Santos, especialista pecuario de la Unidad).

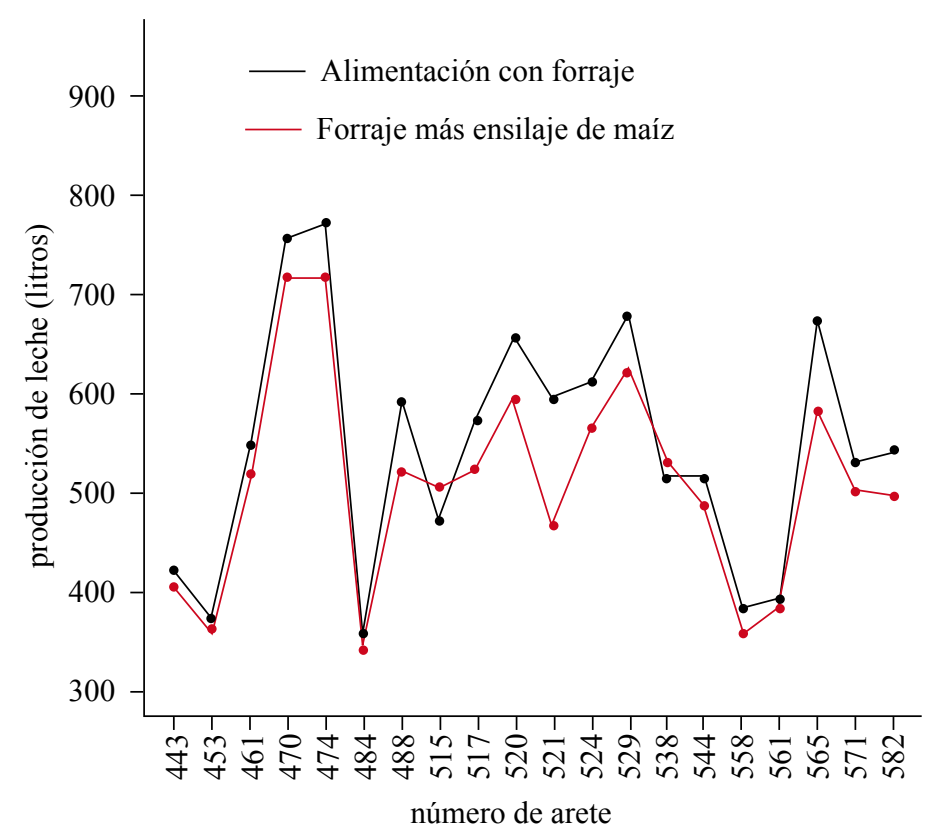

Figura 2: producción de leche de 20 vacas alimentadas con forraje en el mes de julio y con forraje más ensilaje de maíz en el mes de agosto.

- Diferencia de medias entre las muestras pareadas:

V: producción promedio de leche en litros de 20 vacas alimentadas solo con forraje en el mes de julio de 2017.

W: producción promedio de leche en litros de 20 vacas alimentadas con forraje más ensilaje de maíz en el mes de agosto de 2017. 


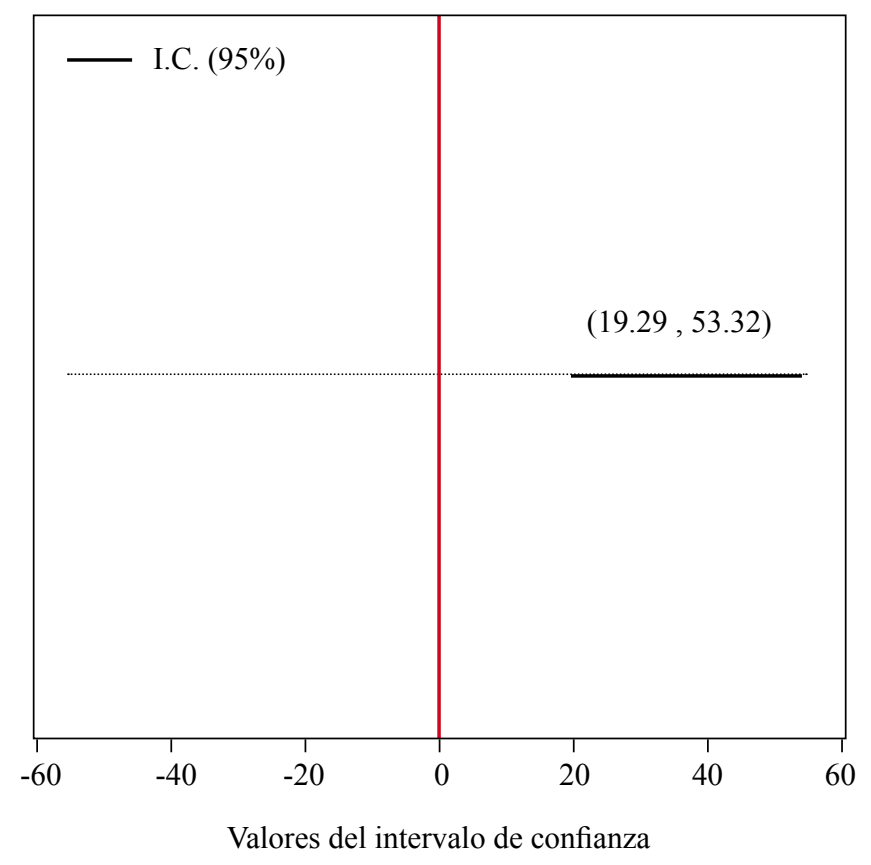

Figura 3: Intervalo de confianza bootstrap al 95\% para la diferencia de medias de las variables, $\mathrm{X}$ e $\mathrm{Y}$.

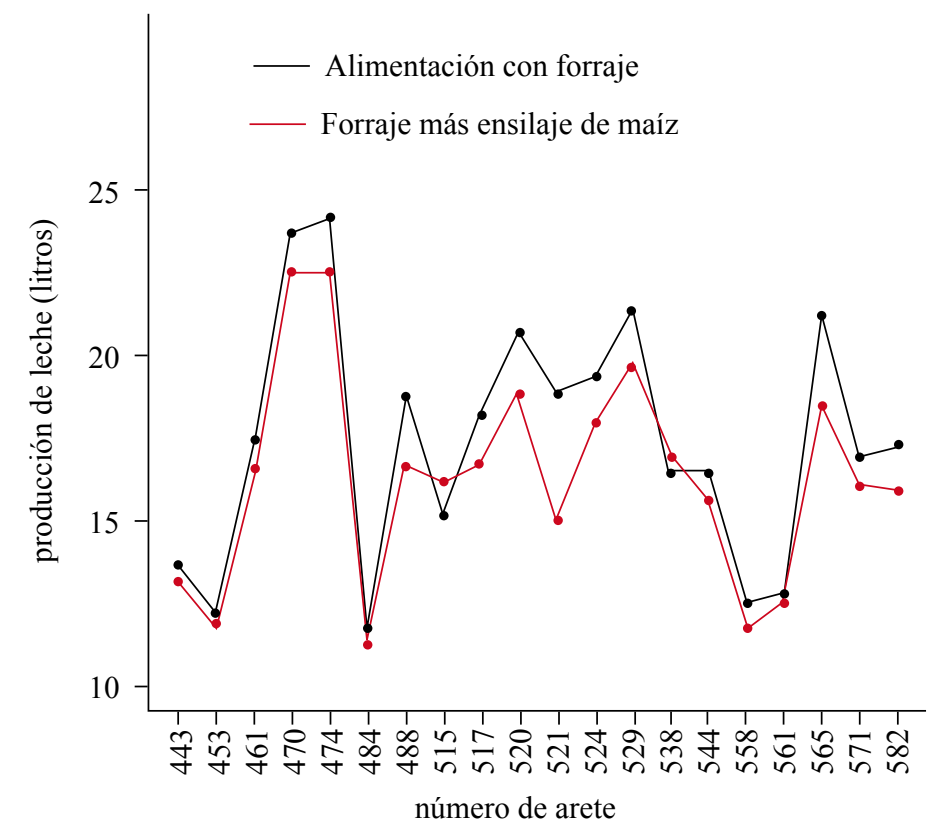

Figura 4: producción promedio de leche de 20 vacas alimentadas con forraje en el mes de julio y con forraje más ensilaje de maíz en el mes de agosto.
Prueba de hipótesis:

$\mathrm{H}_{0}: \mu_{\mathrm{DIF}}=0$ (No existe diferencia significativa entre las medias de las variables $\mathrm{V}$ y W)

$\mathrm{H}_{1}: \mu_{\mathrm{DIF}} \neq 0$ (Sí existe diferencia significativa entre las medias de las variables $\mathrm{V}$ y W).

2. Nivel de significancia $\alpha=0,05$

3. El criterio de aceptar la hipótesis nula es cuando el 0 pertenece al intervalo de confianza Bootstrap, caso contrario la hipótesis nula es rechazada.

4. El intervalo de confianza Bootstrap al 95\% es calculado siguiendo los pasos del algoritmo del método Bootstrap propuesto (con 1000 réplicas):

5. Decisión: En la Figura 5 se observa que el intervalo de confianza Bootstrap no contiene el 0 , por lo que la hipótesis nula se rechaza, es decir que las medias de las variables pareadas $\mathrm{V}$ y W son significativamente diferentes al 95\% de confianza. También se observa que el intervalo de confianza contiene únicamente valores positivos, que significa que la media de producción promedio de leche en vacas alimentadas solo con forraje es significativamente mayor a la media de producción promedio de leche en vacas alimentadas con forraje más ensilaje de maíz.

\section{Estudio de simulación del método propuesto}

Sin pérdida de generalidad, la aplicación del método propuesto se realiza en muestras simuladas de dos normales, con el propósito de comparar con el método tradicional de la t-Student en muestras pareadas $(4,5)$.

En la Tabla 1 se observa que los resultados del método propuesto y del método tradicional de la t-Student son similares. 
La columna Sim. se refiere a 10 veces la simulación de dos muestras normales de tamaño 20, medias, 546 y 509 y desviaciones típicas de 124 y 107. Estas medidas son calculadas de las muestras pareadas de la primera aplicación del método propuesto sobre la producción de leche. Las columnas t-Student y Bootstrap contienen intervalos de confianza al 95\% del estadístico de la media de las diferencias de variables simuladas, ( $\overline{\mathrm{DIF}})$; para la t-Student se calcula el intervalo de la siguiente manera:

$$
\overline{\mathrm{DIF}} \pm 1,96 \frac{\mathrm{S}_{\mathrm{DIF}}}{\sqrt{20}}
$$

donde 1.96 es el cuantil de la normal estándar al 97.5\% y S_DIF es la desviación típica de la variable aleatoria DIF (3).

El intervalo de confianza Bootstrap es calculado con el algoritmo propuesto.

Al comentar al inicio del estudio de simulación que los dos métodos tienen resultados similares se refiere a que los intervalos de las simulaciones de 1 al 9 contienen el 0 , y en la simulación 10 no contiene el cero, esto significa que en las primeras 9 las diferencias de las medias de las muestras no son significativas al $95 \%$ de confianza, mientras que en la 10 la diferencia es significativa.

\section{CONCLUSIONES}

Al finalizar esta investigación de hipótesis inferencial sobre la diferencia de medias para muestras pareadas se concluye:

- El método Bootstrap propuesto es flexible al obviar las condiciones de normalidad y también los tamaños de las muestras pareadas en hipótesis concernientes a la diferencia de sus medias. Esto es una ventaja muy importante para ampliar el campo de aplicación.

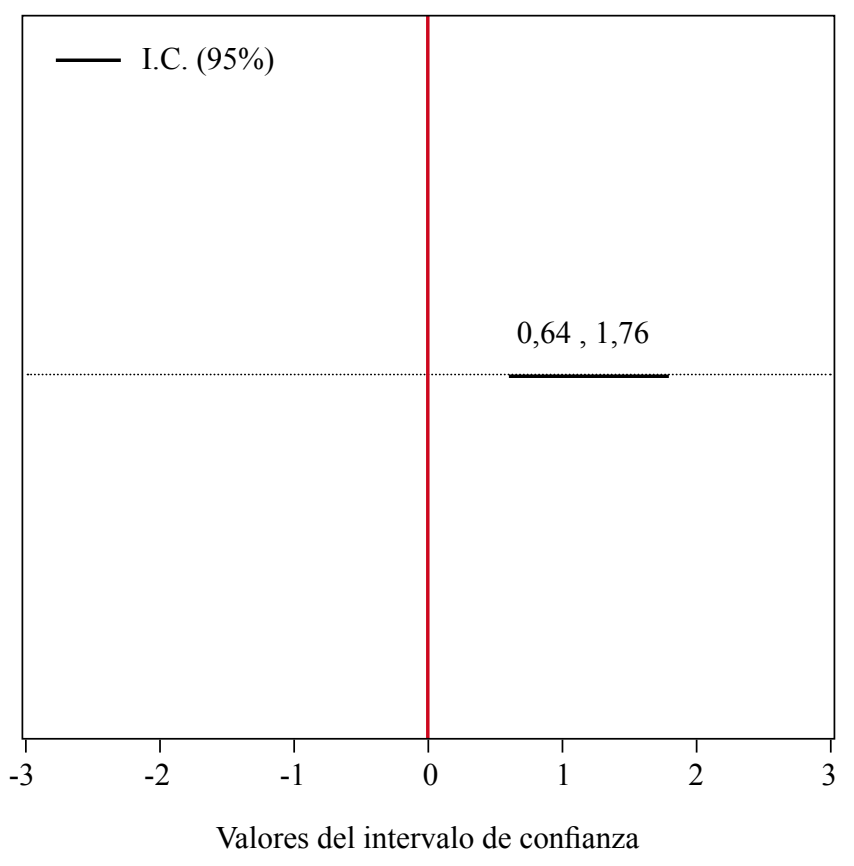

Figura 5. Intervalo de confianza bootstrap al 95\% para la diferencia de medias de las variables, $\mathrm{V}$ y $\mathrm{W}$.

\begin{tabular}{|c|c|c|}
\hline Sim. & t-Student & Bootstrap \\
\hline $\begin{array}{c}\text { Dos muestras } \\
\text { normales }\end{array}$ & $\begin{array}{c}\text { Intervalo de confianza } \\
\text { al 95 } \%\end{array}$ & $\begin{array}{c}\text { Intervalo de confianza } \\
\text { al 95 \% }\end{array}$ \\
\hline 1 & {$[-14,138]$} & {$[-4,150]$} \\
\hline 2 & {$[-27,150]$} & {$[-38,134]$} \\
\hline 3 & {$[-57,79]$} & {$[-50,95]$} \\
\hline 4 & {$[-16,119]$} & {$[-12,114]$} \\
\hline 5 & {$[-120,36]$} & {$[-117,34]$} \\
\hline 6 & {$[-38,149]$} & {$[-33,166]$} \\
\hline 7 & {$[-3,167]$} & {$[-6,171]$} \\
\hline 8 & {$[-87,76]$} & {$[-88,74]$} \\
\hline 9 & {$[-80,75]$} & {$[-84,76]$} \\
\hline 10 & {$[45,177]$} & {$[40,174]$} \\
\hline
\end{tabular}

Tabla 1. intervalos de confianza al $95 \%$ para el estadístico (DIF) por t-Student y el método bootstrap propuesto 
- El método Bootstrap propuesto es una alternativa confiable científicamente para calcular el intervalo de confianza en diferencia de medias para muestras pareadas, y con este rechazar o aceptar la hipótesis nula de forma estadísticamente significativa.

Todos los cálculos de este trabajo se realizaron con herramientas del software estadístico R (6).

\section{AGRADECIMIENTOS}

A los directivos de la Estación Experimental Tunshi, Facultad de Ciencias Pecuarias de la Escuela Superior Politécnica de Chimborazo, Riobamba, Ecuador. Y a la SENESCYT.

1. Davison AC, Hinkley DV. Bootstrap Methods and their Application. Cambridge University Press; 1997.

2. Efron B, Tibshirani R. Bootstrap methods for standard errors, confidence intervals, and other measures of statistical accuracy. Statistical Science 1; 1986.

3. Johnson R. Probabilidad y estadística para ingenieros. Vol 1. 8a ed. México: Pearson educación; 2012.

4. Patrick R. 1982. An extension of Shapiro and Wilk's W test for normality to large samples. Applied Statistics, 31, 115-124.

5. Patrick, R. 1982. Algorithm AS 181: The W test for Normality. Applied Statistics, 31, 176-180.

6. Rizzo M.L. Statistical Computing with R. Chapman\&Hall/CRC; 2008.

7. Wasserman L. All of Statistics. A Concise Course in Statistical Inference. Springer; 2006. 